Curr Opin Struct Biol. 2008 December ; 18(6): 734-740. doi:10.1016/j.sbi.2008.09.010.

\title{
Structural insights into G-protein-coupled receptor activation
}

\author{
William I Weis ${ }^{1,2}$ and Brian K Kobilka ${ }^{1,3}$ \\ ${ }^{1}$ Department of Molecular \& Cellular Physiology, Stanford University School of Medicine, United \\ States \\ 2 Department of Structural Biology, Stanford University School of Medicine, United States \\ 3 Department of Medicine, Stanford University School of Medicine, United States
}

\begin{abstract}
G-protein-coupled receptors (GPCRs) are the largest family of eukaryotic plasma membrane receptors, and are responsible for the majority of cellular responses to external signals. GPCRs share a common architecture comprising seven transmembrane (TM) helices. Binding of an activating ligand enables the receptor to catalyze the exchange of GTP for GDP in a heterotrimeric $\mathrm{G}$ protein. GPCRs are in a conformational equilibrium between inactive and activating states. Crystallographic and spectroscopic studies of the visual pigment rhodopsin and two b-adrenergic receptors have defined some of the conformational changes associated with activation.
\end{abstract}

\section{Introduction}

G-protein-coupled receptors (GPCRs) are a large family of integral membrane proteins responsible for the majority of signal transduction across the eukaryotic cell membrane. GPCRs mediate responses to hormones, neurotransmitters, odorants, and light. As such, they represent important pharmacological targets, and are the targets of somewhere between 25 and $40 \%$ of all drugs currently in use. GPCRs share a common architecture within the membrane consisting of seven transmembrane (TM)-spanning helices, with an extracellular amino terminus and a cytoplasmic carboxy terminus.

Binding of an activating ligand to a GPCR leads to the interaction of the cytoplasmic side of the receptor with a cognate heterotrimeric $\mathrm{G}$ protein. Most GPCRs examined to date catalyze a baseline level of nucleotide exchange by the cognate $\mathrm{G}$ protein in the absence of a ligand. Addition of an activating ligand (an agonist or a partial agonist) increases the exchange activity over this baseline, whereas addition of an inverse agonist reduces activity relative to the unliganded state. Ligands designated antagonists do not affect the baseline exchange activity but prevent the binding of other ligands. These observations suggest that the GPCR exists in a conformational equilibrium in which an activating conformation is sampled sufficiently to produce a baseline level of activity. In this view, agonists and partial

\footnotetext{
Ballesteros-Weinstein numbering is used throughout the text as superscripts to the protein numbering. Within each helix is a single most conserved residue among the class A GPCRs. This residue is designated $x .50$, where $x$ is the number of the transmembrane helix. All other residues on that helix are numbered relative to this conserved position.

Corresponding author: Weis, William I (bill.weis@stanford.edu) and Kobilka, Brian K (kobilka@ @tanford.edu).
} 
agonists shift the equilibrium toward an activating state, whereas inverse agonists shift the equilibrium toward an inactive state. Evidence from both functional and biophysical studies suggests that most GPCRs sample multiple conformations [1].

Given their importance, understanding the mechanism of ligand binding and conformational change associated with GPCR activation has been a long-standing goal. Because of the conformational heterogeneity found in GPCRs, obtaining high-resolution structural information, which requires visualizing one state, has been exceptionally difficult. A very important exception is rhodopsin, the GPCR responsible for sensing light. Unlike GPCRs that bind to diffusible ligands, rhodopsin contains a covalently bound ligand, retinal. In the dark, retinal is in the 11-cis form; this form of the receptor is completely inactive toward its $\mathrm{G}$ protein transducin, a property essential for proper visual physiology. The interaction of retinal with light results in the isomerization of 11-cis retinal to the all-trans form, which triggers conformational changes (detected by a series of spectroscopic intermediates occurring in the femtosecond to millisecond range) that ultimately result in the activated form, metarhodopsin II. Rhodopsin is a member of the largest subgroup of GPCRs, the socalled class A receptors.

The first insights into GPCR structure came from the two-dimensional crystals of rhodopsin $[2,3]$. These structures revealed the general architecture of the seven TM helices. The first three-dimensional crystal structure of dark rhodopsin was reported in 2000 [4] at 2.8 $\AA$, and subsequent structures have been refined to resolutions as high as $2.2 \AA$ [4-9]. These structures revealed a number of features likely to be associated with inactivity such as the ionic lock linking the highly conserved E/DRY sequence found at the cytoplasmic end of TM3 in 70\% of class A GPCRs to TM6 [10] (discussed in more detail below).

The past year has seen remarkable advances in the analysis of GPCR structures. Crystal structures of a cephalopod rhodopsin reveal structural differences that may be related to its specificity for the $\mathrm{G}$ protein $\mathrm{Gq}$ rather than for transducin, the visual $\mathrm{G}$ protein found in the mammalian retina $\left[11^{\bullet}, 12^{\bullet}\right]$. Crystal structures of two b-adrenergic receptors, which are also class A GPCRs, bound to inverse agonists represent the first structures of GPCRs bound to diffusible ligands $\left[13^{\circ}, 14^{\bullet}, 15^{\circ}, 16^{\bullet}, 17^{\circ}\right]$. In addition, the crystal structure of bovine opsin $\left[18^{\circ}\right]$, along with new spectroscopic studies $\left[19^{\circ}\right]$, has provided snapshots along the pathway to activation.

\section{Crystal structures of $\beta$-adrenergic receptors}

Dark rhodopsin is an atypical GPCR in that it is completely inactive toward transducin, suggesting that it is conformationally constrained. In contrast, most GPCRs sample multiple conformations [1], implying that a receptor must be forced into a narrow region of its conformational space in order to be crystallized. Several approaches were taken to overcome the conformational complexity of the $\beta_{1}$-adrenergic and $\beta_{2}$-adrenergic receptors (ARs). In both cases, crystals were obtained with receptors bound to inverse agonists. In the case of the $\beta_{2} \mathrm{AR}$, the inverse agonist carazolol suppresses only half of its basal activity toward the G protein Gs [13** ]. Additional measures to stabilize the $\mathrm{b}_{2} \mathrm{AR}$ focused upon the third intracellular loop (ICL3) that links TM5 and TM6, which is a major site of interaction with 
G proteins. Several lines of evidence suggest that this is a particularly dynamic region of the $\beta_{2}$ AR. Spectroscopic measurements indicate that receptor activation involves movements of TM5 with respect to TM6 [20], and fluorescence lifetime experiments suggest that the cytoplasmic end of TM6 is conformationally heterogeneous [21]. Functional $\beta_{2} \mathrm{AR}$ can be obtained by coexpressing two $\beta_{2} \mathrm{AR}$ fragments split at ICL3 [22], indicating that the intact ICL3 sequence is not needed to stabilize a particular conformation of the receptor. These observations indicate that the conformation of the TM5-ICL3-TM6 region is linked to the equilibrium between inactive and active forms of the receptor. Therefore, stabilizing a single conformation of this region is important for generating a defined state for crystallization.

The first approach to generating and crystallizing a defined conformation of the $\beta_{2} \mathrm{AR}$ was to form a complex with an antibody fragment $\left[13^{\bullet *}, 23\right]$. Most membrane protein crystals are formed by interaction between the water-soluble, extramembranous portions of the molecules, so complexes with Fab or Fv frag ments can provide additional water-soluble surface area that increases the likelihood of lattice formation. An antibody was obtained that recognized a three-dimensional epitope composed of the cytoplasmic ends of TM5 and TM6. A Fab fragment prepared from the antibody bound tightly to the native receptor but did not interfere with ligand binding or with the ability to change conformation upon binding to an agonist [23]. The complex of this Fab with purified receptor was used for crystallization. The native receptor is not very stable in detergents, and diffracting crystals were obtained from lipid bicelles [24,25]. The crystals diffracted anisotropically, with a resolution of $3.4 \AA$ parallel to the plane of the membrane and $3.7 \AA$ in the perpendicular direction $\left[13^{\circ}\right.$ ]. The cytoplasmic side of the receptor and the Fab were reasonably well defined in the electron density map, but the density becomes weaker toward the extracellular side such that none of the extracellular loops were visible, and the ligand-binding site was poorly defined.

The second approach taken to crystallize the $\beta_{2} \mathrm{AR}$ was the replacement of the flexible ICL3 with a small stable protein $\left[14^{* *}, 15^{\circ}\right]$. As with the Fab, the aim was to increase the amount of water-soluble, structured protein surface area for lattice contacts. T4 lysozyme (T4L) was chosen as it has been crystallized under many conditions and because the distance between its $\mathrm{N}$-terminus and its $\mathrm{C}$-terminus is similar to the expected distance between the end of TM5 and the start of TM6. A chimeric $\beta_{2} \mathrm{AR}-\mathrm{T} 4 \mathrm{~L}$ fusion protein bound inverse agonists and antagonists with the same affinity as the wild-type $\beta_{2} \mathrm{AR}$, whereas it bound agonists about twofold to threefold more strongly than the wild-type protein [14**]. Carazolol-bound $\beta_{2} \mathrm{AR}-\mathrm{T} 4 \mathrm{~L}$ was crystallized in a lipidic cubic phase made from monoolein doped with 8$10 \%$ cholesterol $\left[15^{\circ}\right]$. The $\beta_{2} \mathrm{AR}-\mathrm{T} 4 \mathrm{~L}$ crystals diffracted to $2.4 \AA$ resolution, and all the amino acids except for the $22 \mathrm{~N}$-terminal residues and the last $71 \mathrm{C}$-terminal residues could be visualized, as well as the ligand carazolol and several lipid and water molecules. The TM helices of the T4L chimera superimpose closely with those of the wild-type protein in the Fab complex, demonstrating that the structure is not disturbed significantly by the replacement of ICL3 by T4L.

Superposition of the $\beta_{2} \mathrm{AR}$ and dark rhodopsin structures reveals substantial differences in the relative disposition of the TM helices [15*0], particularly in helices III, IV, and V. The overall root-mean-square deviation in the TM helix Ca positions is $2.7 \AA$, which is larger 
than expected based on homology and likely reflects the difference in activation state: $\beta_{2} \mathrm{AR}$ displays G-protein activation activity even when bound to carazolol [13*0], as opposed to the complete inactivity of dark rhodopsin. An important correlate of this difference in activity is shown in Figure 1. In rhodopsin, Glu134 $4^{3.49}$ and Arg $135^{3.50}$ of TM3 form a network of hydrogen bond and ionic interactions with Glu247 $7^{6.30}$ at the cytoplasmic end of TM6. These interactions constitute the ionic lock, which has been proposed to keep dark rhodopsin and other GPCRs in an inactive state [10]. In $\beta_{2} \mathrm{AR}$, TM3 and TM6 are farther apart and the salt bridge between $\operatorname{Arg} 131^{3.50}$ and Glu268 6.30 is absent.

In addition to the broken ionic lock, rhodopsin and $\beta_{2} \mathrm{AR}$ differ substantially on their extracellular faces (Figure 2). In rhodopsin, the second extracellular loop (ECL2) contains two short antiparallel $\mathrm{b}$ strands that pair with two strands from the $\mathrm{N}$-terminus to form a four-stranded $\mathrm{b}$ sheet that buries retinal. In $\beta_{2} \mathrm{AR}$ the $\mathrm{N}$-terminal peptide is disordered, and ECL2 forms an a helix that is locked in position by two disulfide bonds and a number of hydro-phobic packing interactions. The position of the helix leaves the ligand-binding site accessible to the extracellular environment, as might be expected for a receptor that binds diffusible ligands.

The carazolol-binding site lies near the extracellular side of the receptor, and is formed principally by residues from TM helices $3,5,6$, and 7 . The location corresponds to the retinal-binding site of rhodopsin. The observed interactions are consistent with mutational studies that identified residues critical for binding to both antagonists and agonists [14*0]. Also, several residues identified as interacting only with agonists do not contact carazolol. Modeling of the site with the agonist isoproterenol indicated that the structure must rearrange in order for these residues to contact bound agonist $\left[14^{* \bullet}\right.$. The difference between the inverse agonist-bound and the agonist-bound structures will be essential for understanding how agonist binding propagates changes to the cytoplasmic side of the receptor for activating $\mathrm{G}$ proteins.

An interesting feature shared by both rhodopsin and the $\beta_{2} \mathrm{AR}$ is a collection of water molecules that participate in a hydrogen-bonding network extending from the ligand-binding pocket to the cytoplasmic face of the receptor (Figure 3). This network, which includes several of the most highly conserved residues in class A GPCRs, has been proposed to be involved in propagating activating conformational changes in rhodopsin and other GPCRs $[9,26]$.

In a distinct approach to obtaining GPCR crystals, Tate and coworkers used site-directed mutagenesis to produce turkey $\beta_{1} \mathrm{AR}$ variants that are more thermostable than the wild-type receptor $\left[27^{\circ}\right]$. By introducing mutations at six positions in the TM region and deleting residues from the long ICL3 and the extended C-terminus, they produced a protein stable in detergents when bound to antagonists. Crystals diffracting to $2.7 \AA$ resolution were obtained in the detergent octylthioglucoside, from protein bound to the antagonist cyanopindolol $\left[16^{\bullet \bullet}\right.$.

The structures of the turkey $\beta_{1} \mathrm{AR}$ and the human $\beta_{2} \mathrm{AR}$ are overall very similar, including the disulfide-stabilized helix on ECL2 and a broken ionic lock. The two structures are bound 
to structurally similar, nonselective ligands, and the basis for subtype-selective binding in these two receptors is not obvious, as the majority of amino acids making contact with the ligand are identical. Subtype selectivity might involve interactions between the ligands and the more diverse channel leading into the binding pocket $\left[16^{\circ}\right]$. An interesting difference between the structures occurs in ICL2, which is thought to be involved in G-protein activation. In the $\beta_{1} \mathrm{AR}$ structure, this loop contains a short a helix, whereas the $\beta_{2} \mathrm{AR}$ ICL2 adopts a less regular structure.

\section{Activated rhodopsin structure}

Structural and spectroscopic data have provided important insights into the active conformation of GPCRs. EPR and fluorescence studies in which labels are attached to cysteines introduced at various positions demonstrated that TM6 undergoes an outward movement relative to TM3 [28-30]. A more recent EPR method called double-electronelectron resonance (DEER) spectroscopy enabled Hubbel and coworkers to generate a more detailed map of conformational changes involved in the transition of rhodopsin to metarhodopsin II [19*0]. By labeling each of the seven helices in roughly the same plane (near the cytoplasmic surface of the membrane), an outward $5 \AA$ radial movement of TM6 relative to a core composed of TM helices 1-4 appears to be the principal large-scale change associated with the change to an active conformation.

A low-resolution ( $4.15 \AA$ ) crystal structure of a light-activated rhodopsin crystal shows the changes in the relative positions of TM3 and 6 and has a broken lock [31]. Although the spectroscopic signature of the chromophore is similar to that of metarhodopsin II, the relative movements of helices in this case are not as large as expected, and their positions are similar to those of the $\beta$-adrenergic receptors bound to partial inverse agonists. Since the crystals are formed from dark rhodopsin and then bleached, the changes may be restricted by lattice packing forces.

The recent crystal structure of opsin may provide additional insights into the active state of rhodopsin $\left[18^{\circ}\right]$. The apoprotein opsin, which is formed by the hydrolysis of the Schiff base linkage of retinal to the protein after photobleaching, displays at low $\mathrm{pH}$ an infrared spectrum similar to that of metarhodopsin II, suggesting that it is in an active conformation [32]. The crystal structure of low $\mathrm{pH}$ opsin revealed major movements of TM6 consistent with the DEER experiments on metarhodopsin II [19*0. The largest differences between opsin and dark rhodopsin occur at the cytoplasmic end of TM3, TM5, and TM6 (Figure 1). In opsin, the ionic lock is broken, and TM5 and TM6 are now stabilized in new positions by two new interactions involving residues that form the ionic lock in the inactive state. $\mathrm{R} 135^{3.50}$ on TM3, which in the dark forms a salt bridge with E247 $7^{6.30}$ of TM6, now interacts with Y223 $3^{5.58}$ of TM5; E247 $7^{6.30}$ now interacts with K231 5.66 of TM5. It is not known how these structural changes relate to the ability of the activated receptor to interact with its $G$ protein. Although the carazolol-bound $b_{2} A R$ is structurally more similar to rhodopsin, the ionic lock is not formed between Arg $131^{3.50}$ in TM3 and Glu $268^{6.30}$ in TM6. Moreover, the position of TM5 is more similar to TM5 of opsin, and TM6 is slightly displaced toward the position of TM6 in opsin. Thus, the structure of the $\beta_{2} \mathrm{AR}$ appears to be in an intermediate conformation, which may explain its basal activity toward Gs. 
The recent structural studies on GPCRs provide important new insights into the process of receptor activation. However, defining the pathway from agonist binding to $\mathrm{G}$ protein activation will require a combination of crystal structures (including structures of GPCR-G protein complexes), which provide snapshots along the activation pathway, and dynamical information from spectroscopy and molecular dynamics simulations.

\section{Acknowledgments}

The work in the authors' laboratories was supported by grants GM56169 (WIW), NS028471, and GM075811 (BKK).

\section{References and recommended reading}

Papers of particular interest published within the period of review have been highlighted as:

- of special interest

•• of outstanding interest

1. Kobilka BK, Deupi X. Conformational complexity of G-protein-coupled receptors. Trends Pharmacol Sci. 2007; 28:397-406. [PubMed: 17629961]

2. Krebs A, Villa C, Edwards PC, Schertler GF. Characterisation of an improved two-dimensional p22121 crystal from bovine rhodopsin. J Mol Biol. 1998; 282:991-1003. [PubMed: 9753549]

3. Schertler GF, Villa C, Henderson R. Projection structure of rhodopsin. Nature. 1993; 362:770-772. [PubMed: 8469290]

4. Palczewski K, Kumasaka T, Hori T, Behnke CA, Motoshima H, Fox BA, Le Trong I, Teller DC, Okada T, Stenkamp RE, et al. Crystal structure of rhodopsin: a G protein-coupled receptor [see comments]. Science. 2000; 289:739-745. [PubMed: 10926528]

5. Okada T, Le Trong I, Fox BA, Behnke CA, Stenkamp RE, Palczewski K. X-ray diffraction analysis of three-dimensional crystals of bovine rhodopsin obtained from mixed micelles. J Struct Biol. 2000; 130:73-80. [PubMed: 10806093]

6. Okada T, Fujiyoshi Y, Silow M, Navarro J, Landau EM, Shichida Y. Functional role of internal water molecules in rhodopsin revealed by X-ray crystallography. Proc Natl Acad Sci U S A. 2002; 99:5982-5987. [PubMed: 11972040]

7. Teller DC, Okada T, Behnke CA, Palczewski K, Stenkamp RE. Advances in determination of a high-resolution three-dimensional structure of rhodopsin, a model of G-protein-coupled receptors (GPCRs). Biochemistry. 2001; 40:7761-7772. [PubMed: 11425302]

8. Okada T, Sugihara M, Bondar AN, Elstner M, Entel P, Buss V. The retinal conformation and its environment in rhodopsin in light of a new 2.2 ̊ crystal structure. J Mol Biol. 2004; 342:571-583. [PubMed: 15327956]

9. Li J, Edwards PC, Burghammer M, Villa C, Schertler GF. Structure of bovine rhodopsin in a trigonal crystal form. J Mol Biol. 2004; 343:1409-1438. [PubMed: 15491621]

10. Ballesteros JA, Jensen AD, Liapakis G, Rasmussen SG, Shi L, Gether U, Javitch JA. Activation of the beta 2-adrenergic receptor involves disruption of an ionic lock between the cytoplasmic ends of transmembrane segments 3 and 6. J Biol Chem. 2001; 276:29171-29177. [PubMed: 11375997]

11•. Murakami M, Kouyama T. Crystal structure of squid rhodopsin. Nature. 2008; 453:U333-U363. [See annotation to Ref. [12].]

12. Shimamura T, Hiraki K, Takahashi N, Hori T, Ago H, Masuda K, Takio K, Ishiguro M, Miyano M. Crystal structure of squid rhodopsin with intracellularly extended cytoplasmic region. J Biol Chem. 2008; 283:17753-17756. [PubMed: 18463093] [Structures of squid rhodopsin show extensions of TM5 and 6 into the cytoplasm relative to mammalian rhodopsin. This feature may relate to its specificity for $\mathrm{Gq}$ rather than for transducin.]

13••. Rasmussen SGF, Choi HJ, Rosenbaum DM, Kobilka TS, Thian FS, Edwards PC, Burghammer M, Ratnala VRP, Sanishvili R, Fischetti RF, et al. Crystal structure of the human beta(2) 
adrenergic G-protein-coupled receptor. Nature. 2007; 450:U383-U384. [See annotation to Ref. $\left.\left[16^{\bullet \bullet}\right].\right]$

14••. Rosenbaum DM, Cherezov V, Hanson MA, Rasmussen SGF, Thian FS, Kobilka TS, Choi HJ, Yao XJ, Weis WI, Stevens RC, et al. GPCR engineering yields high-resolution structural insights into beta(2)-adrenergic receptor function. Science. 2007; 318:1266-1273. [PubMed: 17962519] [See annotation to Ref. [16 ${ }^{\circ}$ ].]

15••. Cherezov V, Rosenbaum DM, Hanson MA, Rasmussen SGF, Thian FS, Kobilka TS, Choi HJ, Kuhn P, Weis WI, Kobilka BK, et al. High-resolution crystal structure of an engineered human beta(2)-adrenergic G protein-coupled receptor. Science. 2007; 318:1258-1265. [PubMed: 17962520] [See annotation to Ref. [16*0].]

16••. Warne T, Serrano-Vega MJ, Baker JG, Moukhametzianov R, Edwards PC, Henderson R, Leslie AGW, Tate CG, Schertler GFX. Structure of a beta(1)-adrenergic G-protein-coupled receptor.

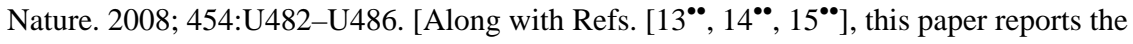
structures of beta-adrenergic receptors bound to inverse agonists, and are the first structures of GPCRs bound to diffusible ligands.]

17•. Hanson MA, Cherezov V, Griffith MT, Roth CB, Jaakola VP, Chien EYT, Velasquez J, Kuhn P, Stevens RC. A specific cholesterol binding site is established by the 2.8 angstrom structure of the human beta(2)-adrenergic receptor. Structure. 2008; 16:897-905. [PubMed: 18547522] [A thermally stabilized $b_{2} \mathrm{AR}-\mathrm{T} 4 \mathrm{~L}$ bound to inverse agonists crystallized from lipidic cubic phase in a lattice different from that of Ref. [15*0]. Comparison of the two structures and sequence analysis suggests a conserved cholesterol-binding pocket among many class A GPCRs.]

18••. Park JH, Scheerer P, Hofmann KP, Choe HW, Ernst OP. Crystal structure of the ligand-free Gprotein-coupled receptor opsin. Nature. 2008; 454:U133-U183. [The crystal structure of low pH opsin, which appears to represent the activating state of this GPCR.]

19••. Altenbach C, Kusnetzow AK, Ernst OP, Hofmann KP, Hubbell WL. High-resolution distance mapping in rhodopsin reveals the pattern of helix movement due to activation. Proc Natl Acad Sci U S A. 2008; 105:7439-7444. [PubMed: 18490656] [A novel EPR method, double-electronelectron resonance (DEER) spectroscopy, is used to map the activating changes in detergentsolubilized rhodopsin.]

20. Ghanouni P, Steenhuis JJ, Farrens DL, Kobilka BK. Agonist-induced conformational changes in the G-protein-coupling domain of the beta 2 adrenergic receptor. Proc Natl Acad Sci U S A. 2001; 98:5997-6002. [PubMed: 11353823]

21. Ghanouni P, Gryczynski Z, Steenhuis JJ, Lee TW, Farrens DL, Lakowicz JR, Kobilka BK. Functionally different agonists induce distinct conformations in the $\mathrm{G}$ protein coupling domain of the beta 2 adrenergic receptor. J Biol Chem. 2001; 276:24433-24436. [PubMed: 11320077]

22. Kobilka BK, Kobilka TS, Daniel K, Regan JW, Caron MG, Lefkowitz RJ. Chimeric alpha 2-,beta 2-adrenergic receptors: delineation of domains involved in effector coupling and ligand binding specificity. Science. 1988; 240:1310-1316. [PubMed: 2836950]

23. Day PW, Rasmussen SGF, Parnot C, Fung JJ, Masood A, Kobilka TS, Yao XJ, Choi HJ, Weis WI, Rohrer DK, et al. A monoclonal antibody for $\mathrm{G}$ protein-coupled receptor crystallography. Nat Methods. 2007; 4:927-929. [PubMed: 17952087]

24. Faham S, Boulting GL, Massey EA, Yohannan S, Yang D, Bowie JU. Crystallization of bacteriorhodopsin from bicelle formulations at room temperature. Protein Sci. 2005; 14:836-840. [PubMed: 15689517]

25. Faham S, Bowie JU. Bicelle crystallization: a new method for crystallizing membrane proteins yields a monomeric bacteriorhodopsin structure. J Mol Biol. 2002; 316:1-6. [PubMed: 11829498]

26. Pardo L, Deupi X, Dolker N, Lopez-Rodriguez ML, Campillo M. The role of internal water molecules in the structure and function of the rhodopsin family of $\mathrm{G}$ protein-coupled receptors. ChemBioChem. 2007; 8:19-24. [PubMed: 17173267]

27-. Serrano-Vega MJ, Magnani F, Shibata Y, Tate CG. Conformational thermostabilization of the beta 1-adrenergic receptor in a detergent-resistant form. Proc Natl Acad Sci U S A. 2008; 105:877-882. [PubMed: 18192400] [This paper describes the screening and characterization of thermostable $\beta_{1}$-adrenergic receptor mutants.] 
28. Farrens DL, Altenbach C, Yang K, Hubbell WL, Khorana HG. Requirement of rigid-body motion of transmembrane helices for light activation of rhodopsin. Science. 1996; 274:768-770. [PubMed: 8864113]

29. Gether U, Lin S, Ghanouni P, Ballesteros JA, Weinstein H, Kobilka BK. Agonists induce conformational changes in transmembrane domains III and VI of the beta2 adrenoceptor. EMBO J. 1997; 16:6737-6747. [PubMed: 9362488]

30. Yao X, Parnot C, Deupi X, Ratnala VR, Swaminath G, Farrens D, Kobilka B. Coupling ligand structure to specific conformational switches in the beta2-adrenoceptor. Nat Chem Biol. 2006; 2:417-422. [PubMed: 16799554]

31. Salom D, Lodowski DT, Stenkamp RE, Le Trong I, Golczak M, Jastrzebska B, Harris T, Ballesteros JA, Palczewski K. Crystal structure of a photoactivated deprotonated intermediate of rhodopsin. Proc Natl Acad Sci U S A. 2006; 103:16123-16128. [PubMed: 17060607]

32. Vogel R, Siebert F. Conformations of the active and inactive states of opsin. J Biol Chem. 2001; 276:38487-38493. [PubMed: 11502747] 


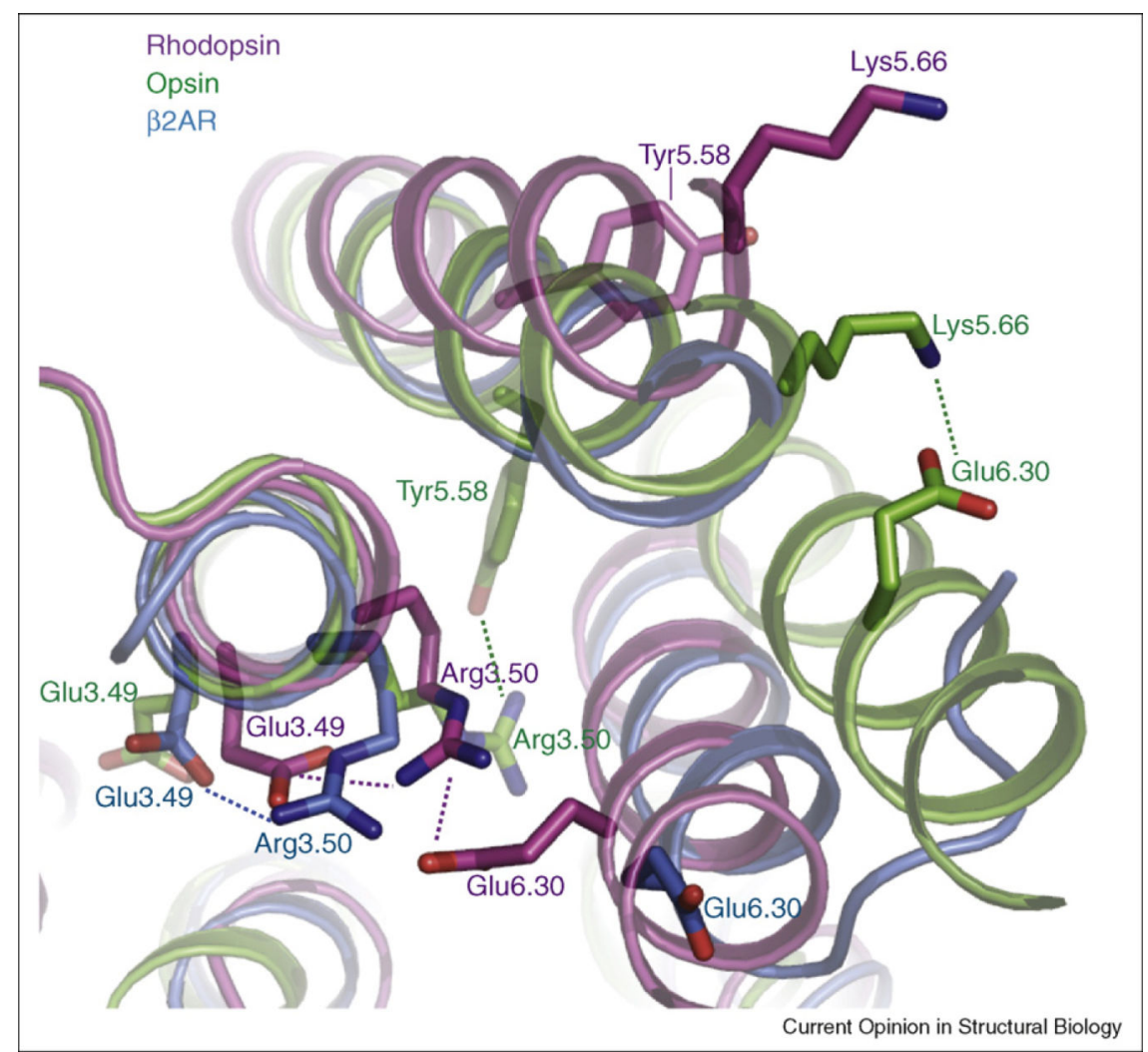

Figure 1.

An overlay of dark rhodopsin (purple; PDB 1gzm), low pH opsin (green; PDB 3cap), and carazolol-bound $\beta_{2} \mathrm{AR}-\mathrm{T} 4 \mathrm{~L}$ (blue; PDB 2rh1) in the vicinity of the ionic lock. In dark rhodopsin, Arg135 3.50 in the conserved D/ERY sequence near the cytoplasmic end of TM3 forms a salt bridge with Glu2476.30 at the cytoplasmic end of TM6. Arg 3.50 is further stabilized by a salt bridge to the preceding conserved acid at position 3.49 in both rhodopsin and $\beta_{2}$ AR. In opsin, Arg135 3.50 interacts with Tyr223 5.58 in TM5, and Glu247 6.30 forms a salt bridge with Lys $231^{5.66}$. The homologous ionic lock residues Arg $131^{3.50}$ and Glu268 6.30 from the $\beta_{2} \mathrm{AR}$ are also shown. The amino acids are numbered using the BallesterosWeinstein system. Within each helix is a single most conserved residue among the class A GPCRs. This residue is designated $x .50$, where $x$ is the number of the transmembrane helix. All other residues on that helix are numbered relative to this conserved position. 


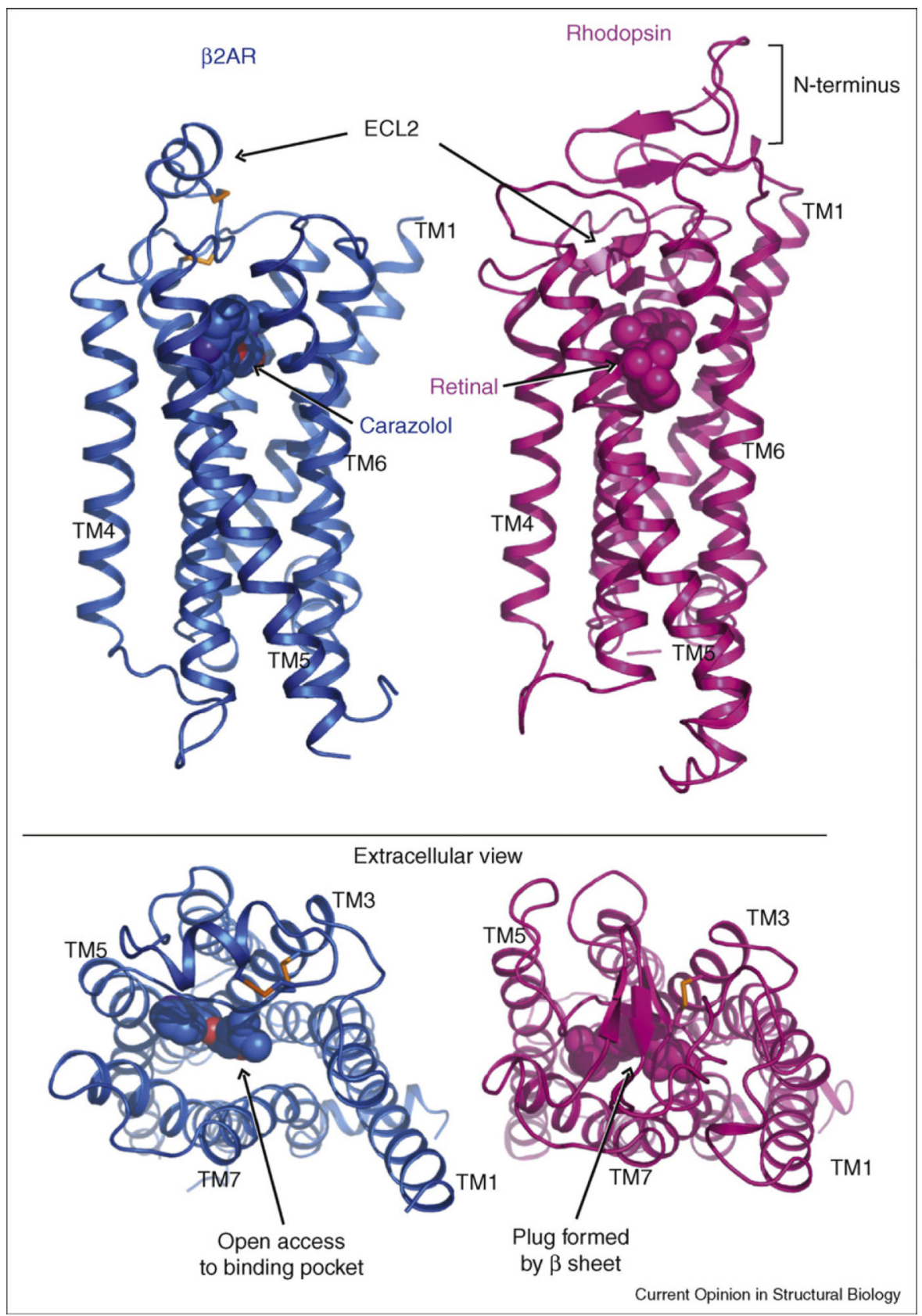

Figure 2.

Comparison of rhodopsin (purple; PDB 1gzm) and carazolol-bound $\beta_{2} \mathrm{AR}-\mathrm{T} 4 \mathrm{~L}$ (blue; PDB 2rh1). Extracellular loop 2 (ECL2) of rhodopsin forms a lid over the retinal-binding pocket, whereas the position of ECL2 of the $\beta_{2} \mathrm{AR}$ allows relatively free access to the carazololbinding pocket. 


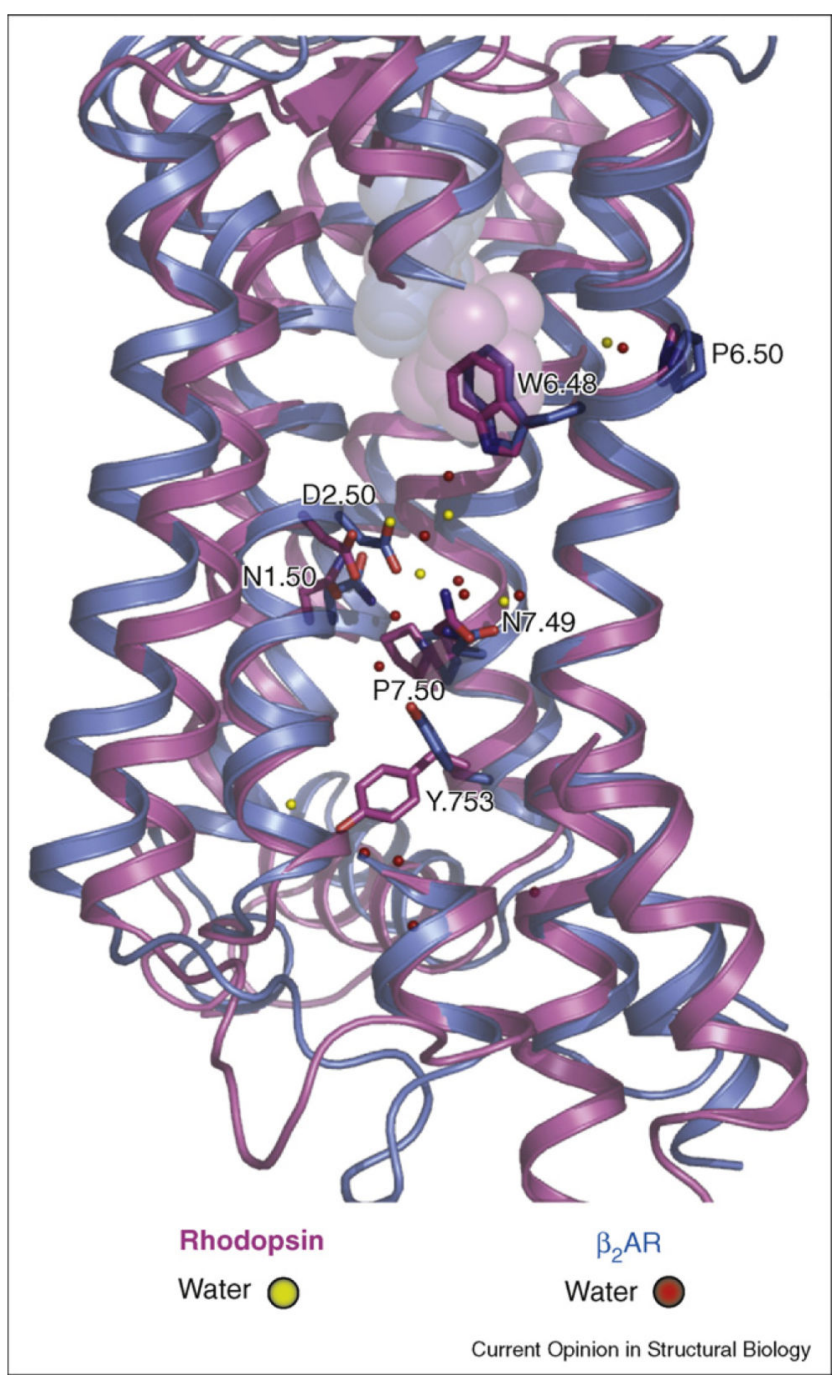

Figure 3.

Superimposed structures of rhodopsin (purple; PDB 1gzm) and carazolol-bound $\beta_{2} \mathrm{AR}-\mathrm{T} 4 \mathrm{~L}$ (blue; PDB 2rh1) highlighting water molecules that form a hydrogen-bond network with conserved amino acids. Atoms of carazolol and retinal are shown as spheres. The amino acids are numbered using the Ballesteros-Weinstein system (see Figure 1). 